\title{
Importando la torre al barrio. Procesos de densificación intensiva en la periferia metropolitana de Buenos Aires
}

Moving the towers into the neighborhoods Intensive densification processes in the outskirts of Buenos Aires

Importando a torre para o bairro Processos de densificação intensiva na periferia metropolitana de Buenos Aires Guillermo Tella (dir.) ${ }^{*}$, Estela Cañellas
Martín Muñoz
y Daniela Natale
Instituto del Conurbano, Universidad Nacional de General Sarmiento
Argentina

\section{Resumen}

La explotación intensiva de áreas urbanas de baja ocupación constituye un vector que permite indagar acerca de las lógicas que operan en los procesos de transformación de la ciudad consolidada y en la identificación de actores y políticas urbanas involucradas. Este artículo trata de caracterizar los cambios urbanos recientes, examinando los procesos de toma de decisiones en torno de la construcción de edificios en torre a partir de la mirada sobre un caso testigo. Y apunta a contribuir a la comprensión de los mecanismos de producción y gestión urbana, los intereses en juego y la red de actores que habilitan una densificación intensiva.

Palabras claves: políticas urbanas, mecanismos de producción, toma de decisiones, densificación intensiva, edificios en torre.

* Guillermo Tella:Arquitecto, Doctorado en Ordenamiento Territorial.Universidad de Buenos Aires. <gtella@ungs.edu.ar>

** Estela Cañellas: Licenciada en Urbanismo y Diplomada en Ciencias Sociales, Universidad Nacional de General Sarmiento. Posgrados en Derecho Urbanístico,Acceso al Suelo Urbano para Sectores Populares en Argentina y Gestión del Suelo en Grandes Proyectos Urbanos. <estelacaniellas@gmail.com>

*** Martín Maximiliano Muñoz:Traductor público en idioma inglés, Pontificia Universidad Católica Argentina Santa María de los Buenos Aires. <martinmaxmunoz@gmail.com>

***** Daniela Natale: Licenciada en Urbanismo y Diplomada en Administración, Universidad Nacional de General Sarmiento. Maestría en Gestión Ambiental del Desarrollo Urbano, Universidad Nacional de Mar del Plata. <dnnatale@ungs.edu.ar> 


\section{Abstract}

The intensive exploitation of low-density land occupation areas is a vector which allows inquiring into the logics taking part in the processes of transformation of the consolidated city and into the identification of actors and urban policies involved. This article is an attempt to characterize recent urban changes by looking into the decision-making processes around the construction of high-rising buildings from the analysis of a leading case, and aims to contribute to the comprehension of the mechanisms of urban production and management, the interests involved and the network of agents which enables an intensive densification.

Keywords: urban policies, mechanisms of production, decision-making, intensive densification, high-rising buildings.

\section{Resumo}

A exploração intensiva de áreas urbanas de baixa ocupação constitui um vetor que permite indagar sobre as lógicas que operam os processos de transformação da cidade consolidada e na identificação de atores e políticas urbanas envolvidas. Este artigo tenta caracterizar as mudanças urbanas recentes, examinando os processos de tomada de decisões sobre a construção de arranha-céus a partir do ponto de vista de um caso real. E propõe contribuir com a compreensão dos mecanismos de produção e gestão urbana, com os interesses em jogo e com a rede de atores que possibilitam uma densificação intensiva.

Palavras-chave: políticas urbanas, mecanismos de produção, tomada de decisões, densificação intensiva, arranha-céus.

\section{Introducción}

La explotación intensiva de áreas urbanas de baja ocupación constituye un vector que permite indagar acerca de las lógicas que operan en la construcción de la ciudad. Muchos de los nuevos productos inmobiliarios, como las "torres", se gestan en el contexto de un proceso de demandas de un mercado dirigido a sectores medio-altos que buscan "refugio" dentro de la ciudad consolidada.

El impacto de esos edificios no es menor, pues generan la ruptura de las estructuras urbanas sedimentadas a través del tiempo y la degradación cualitativa de las preexistencias.Asimismo, los vecinos hacen oír sus voces de rechazo al fenómeno instalado. ¿Por qué se construyen las torres? ¿Quiénes están a favor, quiénes en contra? ¿Cuál es su impacto en la ciudad construida? Estas son algunas de las interrogantes que se intentan responder. Desde esta perspectiva, el trabajo da cuenta de los procesos de transformación de la ciudad consolidada y de la identificación de las políticas urbanas que operan. 
De modo que se tratan de caracterizar los cambios urbanos recientes, examinando los procesos de toma de decisiones -los actores públicos y privados, los marcos institucionales e instrumentos- en torno a la construcción de las torres. A partir de la mirada sobre un caso testigo, se apunta a contribuir a la comprensión de los mecanismos de producción y de gestión, los intereses en juego y la red de actores que habilitan una densificación intensiva.

\section{Acerca de la cuestión post-urbana}

Buenos Aires es una aglomeración urbana que supera los 13 millones de habitantes y que pertenece a espacios económicos no centrales. Exhibe un fuerte contraste entre centro y periferia, lo que pone de manifiesto la presencia de determinados patrones característicos de una inserción aguda en un sistema global de ciudades.

Así como se refleja en estudios recientes sobre la evolución de la dinámica urbana en la Región Metropolitana de Buenos Aires, este patrón muestra la destrucción de tejidos fabriles e industriales, la construcción de ámbitos destinados a la expansión de la economía financiera, el desarrollo de nuevas tipologías edilicias residenciales, nuevas formas de distribución comercial sustentadas en el uso del automóvil particular, la aparición de nuevas modalidades de comercialización del ocio en las periferias, la inversión en estructura viaria para dinamizar los desplazamientos (De Mattos, 200 I;Torres, 200 I y Ciccolella, 1999). Como contrapartida, se percibe el incremento de los asentamientos precarios y de los niveles de violencia urbana y de la fragmentación socio-territorial.

Asimismo, en la última década se ha evidenciado un crecimiento dicotómico como resultado de una modernización acelerada, tardía respecto de sus modelos centrales de referencia (Tella, 200I). Por un lado muestra una acentuada concentración de la riqueza en determinados sectores sociales y por otro, un empobrecimiento extremo, ante la incapacidad de absorción de fuerzas de trabajo por parte de la industria urbana (Schneier-Madanes, 1998).

De este proceso diferencial se advierte que, mientras una parte del territorio metropolitano es objeto de inversión en equipamientos e infraestructuras de todo tipo -exhibiendo los efectos de una economía urbana integrada al sistema global-, la otra parte no es convocada para ello. Espacialmente, se evidencia en el surgimiento de espacios estratégicos reestructurados, debido a una fuerte concentración de inversiones de capital, contrapuestos a la persistencia de amplias áreas residuales, ámbitos en los que se manifiesta un cierto abandono debido a que no resultan de su interés (Tella, 2007).

Esta situación, entonces, se traduce en la formación de verdaderos enclaves urbanos, con aspectos más agudos y de naturaleza más crítica respecto de aquellos atribuidos a las metrópolis de países centrales (Borja y Castells, 1998). Efectivamente, los cambios mundiales en la economía y su instalación en el contexto local se encuentran en la base de la evolución reciente de la región metropolitana de Buenos Aires. Estos se traducen en la aparición de nuevas formas residenciales, de nuevos patrones de consumo así como de nuevos desarrollos del terciario avanzado. 
La generación de espacios estratégicos se produce en Buenos Aires de manera diferenciada y con características específicas, a través de un equipamiento selectivo del territorio y del incremento de sus desigualdades. Las transformaciones territoriales que hoy presenta tienen aspectos comunes con los observados en otras ciudades de su talla, sin embargo, las características propias de su evolución socioespacial hacen que su impacto sea diferente. Esto se debe a que se agudizan los cortes existentes en el interior del amplio abanico de sus tradicionales clases medias (Ciccolella, 1999 y Torres, 200 I).

Con lo cual, muy recientemente tienen lugar, por una parte, los procesos residenciales que establecen enclaves de alto nivel sobre la extrema periferia, acompañados por la expansión también periférica del terciario avanzado. Por otra, se generan procesos de deterioro central conducentes a la formación de guetos. Como respuesta, surge entonces el concepto de "urbanización cerrada" para conciliar distintos valores. Estos son: tierra barata (que aprovecha terrenos de grandes dimensiones), accesibilidad (debido a su proximidad a las autopistas), valores paisajísticos (mediante una evocación a la naturaleza) y seguridad (a través del recurso de la muralla y de la vigilancia privada).

Buenos Aires se encuentra inmersa en un proceso de reconfiguración territorial caracterizado, en parte, por la difusión de un entorno urbano extenso y ajerárquico, fragmentado y discontinuo. Esta reconfiguración conduce a la deconstrucción de los clásicos conceptos de "centro" y de "suburbio" frente al surgimiento de centralidades de nuevo tipo. Por otro lado, la tendencia hacia una economía de servicios que induce a una gran dispersión espacial de las actividades, impulsada por las innovaciones tecnológicas que desplazan las fuentes de trabajo de las áreas centrales hacia los bordes metropolitanos.

\section{Una recualificación socioterritorial}

Buenos Aires se ha caracterizado históricamente por su fuerte estructura monocéntrica, de manera que su downtown tuvo una supremacía irrefutable respecto del segundo nivel de centros de la aglomeración. Asimismo, los asentamientos residenciales surgieron y se consolidaron a través del tiempo en estrecha articulación con el trazado ferroviario. De manera que la última década detonó fuertes procesos de reconversión económica e hiperurbanización acelerada que produjeron de manera simultánea una expansión y una recualificación socioterritoriales. (Tella, 2003).

Del conjunto de efectos visibles es preciso subrayar, con mayor predominancia por un lado, la generación de nuevas centralidades periféricas que impactaron contra los bordes de la ciudad construida. La materialización se posibilitó a partir de una descentralización comercial, principalmente sobre los vacíos intersticiales de la segunda corona, por el gran repliegue industrial, y por la desconcentración residencial, situada sobre la extrema periferia e impulsada por el desarrollo de las highways. Esta situación encubre impensables proyecciones a futuro (Ciccolella, 1999 y Torres, 200I).

Por otro lado, un proceso de recentralización de centralidades existentes, para permitir la expansión de actividades administrativas y financieras a partir de una creciente participación de capitales transnacionales. Con estos aportes se "modernizó" 
la imagen del antiguo centro con arquitecturas emblemáticas de fuerte impacto visual y económico (cuyo puntapié inicial fue dado por la reconversión de Puerto Madero).

Ambos procesos se llevaron a cabo de forma espontánea, por iniciativa privada, sin responder a ningún tipo de estrategia regional y en el marco de un laissez-faire territorial. El primero, sobre la extrema periferia en colisión con los bordes consolidados como suburbanización popular. El segundo, llenando los vacíos intersticiales dejados por el anterior avance de la ciudad entre los ejes de expansión.

Su dosificación dio como resultado una nueva fisonomía urbana caracterizada por: (a) la dispersión periférica, (b) la difusión de subcentralidades, (c) la fragmentación socio-territorial y (d) la discontinuidad de tejidos, como principales factores de motorización de las transformaciones territoriales recientes (Tella, 2005).

Debe subrayarse, entonces, que se trata de un proceso brusco y acelerado de modernización, en el que se llevaron a cabo actuaciones en forma extrema y tardía, que en los países centrales se desarrollaron paulatina y morigeradamente a lo largo de varias décadas. En cambio, aquí en sólo diez años, se materializó esta situación dicotómica de periferización de áreas centrales y de centralización de áreas periféricas. El proceso fue el resultado de un agudo proceso que encubrió el desvanecimiento industrial y la descentralización comercial junto con desconcentraciones residenciales espontáneas. Esta lógica de urbanización, a través de enclaves de alto nivel, comenzó prontamente a evidenciar conflictos derivados de la dispersión.

Este proceso no ha alcanzado aún su punto de inflexión. Por lo tanto, la situación planteada, por un lado, abre numerosas e importantes interrogantes en relación con la evolución futura de Buenos Aires. Por otro, manifiesta con claridad la presencia de un fenómeno urbano en expansión con consecuencias trascendentes e irreversibles sobre la estructura metropolitana. Uno de ellos lo constituye la densificación intensiva sobre áreas de baja ocupación, que derivan en conflictos de articulación con visibles expresiones territoriales.

\section{Los procesos de densificación intensiva}

La introducción de la tipología edilicia "torre", como forma de densificar la ciudad, no es cualidad distintiva de esta época. Sus antecedentes se remontan a varias décadas. Un hito determinante lo constituye la entrada en el debate urbanístico del Movimiento moderno, que daba cuenta de la necesidad de resolver problemas habitacionales. Con la Carta de Atenas (1933) se instala la idea de ciudad ordenada en áreas funcionales claramente definidas mediante un modelo analítico de abordaje basado en una categorización rígida de las funciones urbanas: habitar, trabajar, recrearse y circular. En este modelo la "verticalización" era un eje fundamental.

En Buenos Aires, si bien los procesos de sustitución intensiva han comenzado a resignificarse en la última década, la torre tampoco representa un fenómeno reciente. La década de 1930 puede tomarse como punto de partida para el crecimiento vertical de la capital, tomando como modelo a Nueva York, tras la inauguración del 
famoso Empire State Building (1931). Claros ejemplos de la influencia local del Movimiento moderno lo constituyen los edificios Safico (1933), Comega (1933) y Kavanagh (1935), en el centro de la ciudad.

Tras la Ley de Propiedad Horizontal (1948) se impulsó una densificación mediante el completamiento del tejido con edificios entre medianeras. Hacia 1960 resurgen los grandes conjuntos habitacionales en la ciudad consolidada: Los conjuntos de Catalinas Sur (1962), General Savio (1969); Soldati (1972) y Luis Piedrabuena (1974) constituyen algunos de los principales exponentes.

Otro hito importante se produjo en la última década, con nuevos procesos de sustitución intensiva que tuvo alta visibilidad en varios barrios de Buenos Aires y su espacio metropolitano. El proceso comenzó en 2003 con el boom de la construcción y se posicionó en el centro del debate tres años más tarde en los barrios de Caballito, Palermo, Núñez,Villa Urquiza,Villa Pueyrredón y Vicente López.Allí, los vecinos alzaron sus voces contra las transformaciones territoriales que estaban sufriendo con la irrupción de edificios en torre sobre tejidos residenciales de baja y (o) media densidad.

Caballito, por ejemplo, es un caso paradigmático de este proceso de sustitución intensiva. En un radio de 50 manzanas se localizaron más de 100 nuevos edificios de departamentos a estrenar. En un año, el precio promedio de venta por metro cuadrado de estas unidades se incrementó en un 25\%, pasando de mil 350 a mil 690 dólares. Hacia fines de 2006, ante los reclamos vecinales, se puso un freno parcial a la emisión de permisos para construir torres en Buenos Aires, con el propósito de analizar los impactos de este tipo de densificación en relación con la provisión de servicios públicos, de espacios verdes disponibles, etc.

Una situación similar se produjo en torno a la costa de Vicente López. Tras la refuncionalización de toda esa área degradada, las zonas aledañas se convirtieron en oportunidad para el desarrollo inmobiliario. Los reclamos de las organizaciones vecinales contra las excepciones a la normativa motivaron la elaboración de un esquema director para ese frente costero. Hoy se encuentran aprobados y en ejecución más de 20 edificios en torre $y$ un megaproyecto que incluye hoteles, shopping, complejo multicine y centro de convenciones.

Pujas y tensiones están instaladas en el seno mismo de una metrópolis como Buenos Aires, que desde hace un par de décadas acrecienta su tendencia a la segregación social y a la privatización del espacio público. Este nuevo sistema de relaciones en parte se expresa en nuevas formas de residencialidad: las torres, que privilegian valores como la seguridad, la exclusividad y la "naturaleza" para la micro-comunidad que alberga y que, en contraposición con las urbanizaciones cerradas, permiten gozar de dichos privilegios, sin perder los beneficios de vivir en la ciudad.

Este modelo, que busca preservar física y simbólicamente a unos de otros, genera grandes tensiones y disputas sobre el mercado del suelo. La periferia fue, históricamente, el área de residencia de los sectores medios y bajos de la población; hoy se presenta como territorio de conflictos. Esta ruptura de patrones tendenciales de crecimiento fomenta las asimetrías socioespaciales, bunkerizando ciertos sectores de borde y pauperizando al extremo otros. 
En consecuencia, más allá de las particularidades de cada caso, es posible observar cómo el Estado en estos procesos ha abandonado su papel como agente directo de canalización de las demandas sociales insatisfechas, para convertirse en facilitador de las actividades del mercado. $Y$ esto se realiza mediante la provisión de las herramientas necesarias para que las inversiones privadas prosperen.

Contemporáneamente, la crisis del modelo cientificista y tecnocrático del urbanismo devino en la búsqueda y el desarrollo de nuevas teorías que abrieran un sendero de solución práctica a los desafíos emergentes de la aplicación del modelo mencionado. Dentro de ese ámbito, surgen nuevos paradigmas de gestión de lo urbano, como la gobernanza, y que, con inéditas aproximaciones a la gestión de redes de actores, brindan nuevas herramientas al servicio de las administraciones públicas, en especial de los gobiernos locales. Sin embargo, la puesta en práctica supone la superposición, entre convivencia y conflicto, de ambos modelos de gestión.

En este marco contextual se inserta el caso del municipio de San Miguel, en el conurbano bonaerense como caso testigo de los efectos de este proceso en un área central de la periferia de una metrópolis.

\section{La evaluación del modelo de desarrollo}

La ciudad de San Miguel es la cabecera del municipio homónimo, situado en el noroeste del área metropolitana de Buenos Aires.' Cuenta con 253 mil habitantes, distribuidos de manera dispar y focalizada, y representa menos del $3 \%$ de la población de la metrópolis (INDEC, 200I). La importancia de esta ciudad se debe, en primer lugar, a su papel histórico como cabecera de partido, concentrando más del $60 \%$ de su población total. Además, porque se caracteriza "por su ventajosa topografía, que concilió ventajas de accesibilidad con otras de tipo estético" lo que configura "un planteo inicial sano" (Randle, 1977, p. 160).

Como subcentro regional, se posiciona como centralidad regional en la corona de ciudades definidas por el Río Reconquista (San Fernando, Tigre, San Miguel, Moreno, Merlo) (Atlas Ambiental de Buenos Aires, 2009) (ver ilustración I).

En San Miguel se encuentra el área de estudio, conformando un polígono de 16 manzanas delimitado por la Av. Pdte. Perón y las calles San José, Serrano y Rodríguez Peña.Asimismo, se determinó un área de influencia de cuatro manzanas a la redonda desde su límite externo con el fin de considerar los servicios y la conectividad que proveen al área de estudio.

I Con relación a la definición de Área Metropolitana de Buenos Aires, cf. Diagnóstico ambiental preliminar del Partido de San Miguel (Miño, Borello y Alsina, 2007) y ¿Qué es el Gran Buenos Aires? (INDEC, 2003). 


\section{Ilustración I}

Localización del partido de San Miguel en el Área Metropolitana de Buenos Aires.

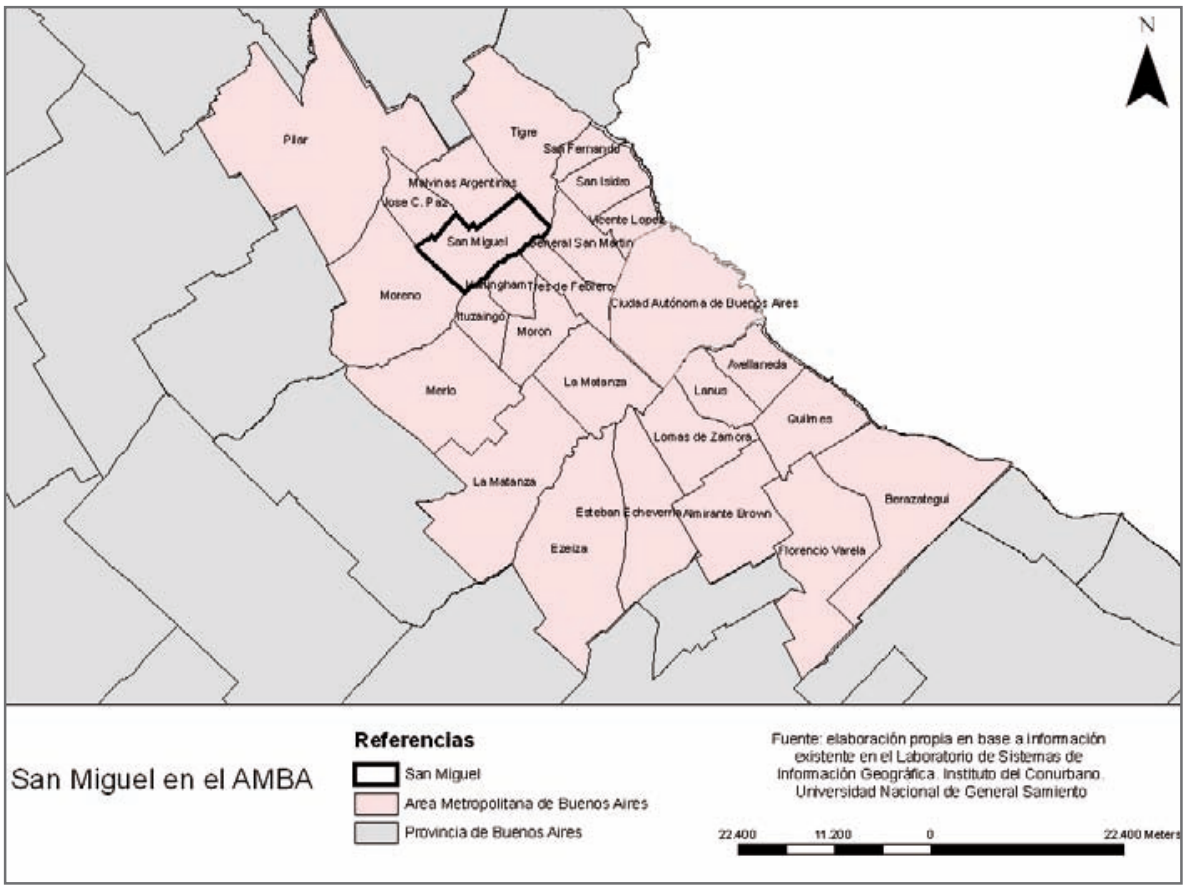

Fuente: Elaboración propia en base a información del LabSIG-UNGS.

Las motivaciones que llevaron a la selección de esta área de estudio, responden a varios criterios: intensa concentración de los edificios en "torre", altos indicadores urbanísticos en la normativa, predominancia de un tejido residencial de baja densidad, importante conectividad con centralidades del conurbano, articulación con los principales ejes articuladores, proximidad a núcleos comerciales y a servicios públicos, y "balconeo" sin obstáculos de las torres como capital paisajístico (Ver imagen 2).

El área de estudio presenta un tejido originariamente de tipo residencial de baja densidad con un cierto uso mixto comercial que se acrecienta sobre los ejes viales del partido (Av. Pdte. Perón y calle San José). No existen espacios verdes públicos pero, sin embargo, el arbolado de mediana estatura en las aceras dota al paisaje urbano de un ambiente agradable y lo embellece, así como los jardines al frente de las casas. A esto se suman las fachadas que presentan un aspecto cuidado y de buen mantenimiento. También cuenta con servicio de barrido y limpieza por parte de una

2 Se refiere a importantes espacios verdes pasivos, tales como Campo de Mayo o la frondosa arboleda de la zona residencial de baja densidad de Bella Vista y las quintas de San Miguel y Muñiz. La cuestión del capital paisajistico siempre ha sido gravitante en la elección de estas localidades para su ocupación y desarrollo poblacional y urbanístico. (Randle, 1977, pp. I58-160). 
empresa recolectora de residuos, que permite mantener en buenas condiciones los sumideros y la escorrentía de aguas superficiales a los pluviales (Covelia, 2009).

Ilustración 2

Delimitación del área de estudio y del área de influencia, con identificación de los edificios en torre.

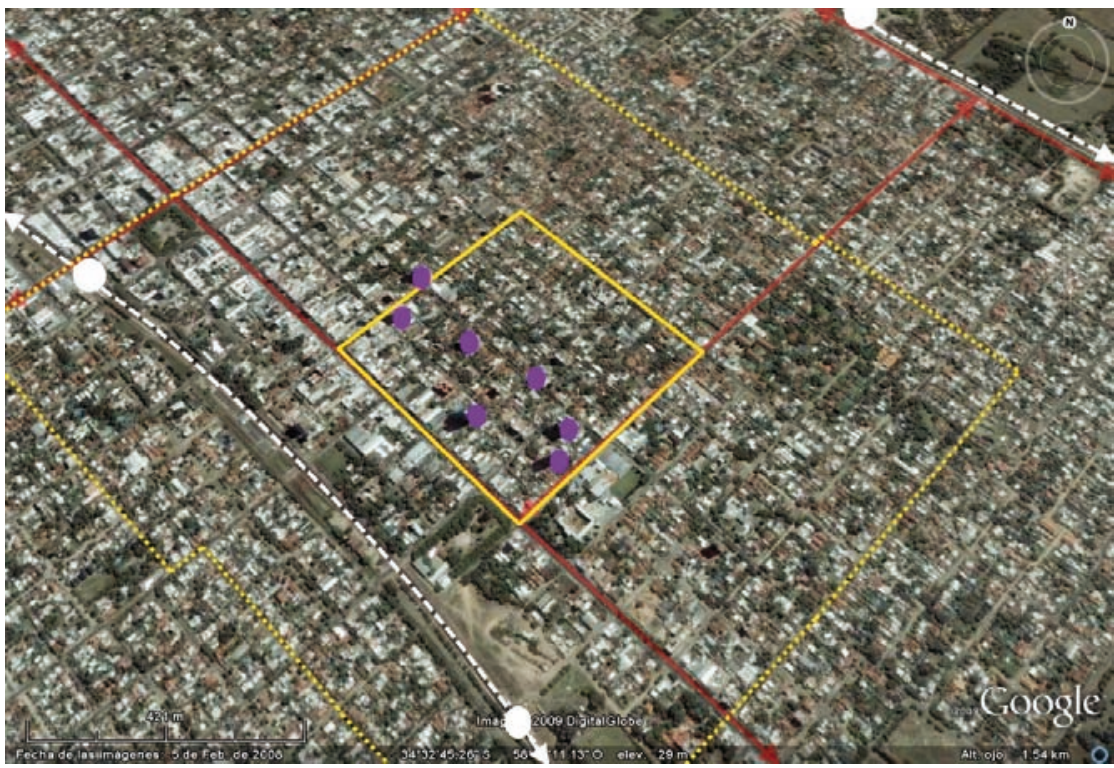

Fuente: Elaboración propia sobre imagenes satelitales tomadas de Google Earth.

\section{Las cualidades del área de estudio}

En los últimos años en el área se han realizado inversiones municipales en: señalización de calles (Av. Pdte. Perón, Sgto. Cabral, Serrano y San José), semaforización de determinados cruces (Serrano y San José, Maestro Ángel D’Elía y San José) y recambio de semáforos por otros con asistencia para el cruce de no videntes (sobre los cruces de la Av. Pdte. Perón). Mucho de ello se concretó gracias a la implementación de la política de presupuesto participativo (Municipalidad de San Miguel, 2009).

Estos detalles -sumados a la cercanía de importantes espacios verdes, a los servicios de transporte ferroviario y automotor de pasajeros y a la provisión de servicios sociales y de redes- involucran en su conjunto el sostén gubernamental de un tejido de condiciones propicias para el desarrollo de una plusvalía urbana asequible sólo con asentamiento de clases sociales de ingresos medio-altos. Este hecho contrasta fuertemente con las inversiones que históricamente se pueden haber prodigado en otros sectores más apartados del centro tradicional del partido. Además, estos mismos criterios de selección y cualidades del entorno sirven, en definitiva, al 
mercado inmobiliario para elegir esta zona y capitalizarlo en el valor de venta de los nuevos metros cuadrados construidos en el producto final.

Con la puesta en vigencia del Decreto-Ley 89/2/77 y la ordenanza que la ponía en práctica a nivel municipal (448/79), el modelo zonificado de ciudad prospectiva trazado por ambas legislaciones se comenzó a plasmar sobre el territorio recién a partir de fines de la década de 1990. En este momento comienza la instalación de edificios en torre dentro de la zona denominada “Microcentro". Los límites del área son las vías del ferrocarril San Martín, las calles Int. Irigoin, Serrano, Luis María Argüero, César Malnatti, Julio A. Roca,Vicente López, San Luis, Rodríguez Peña, Serrano, Haedo, Maestro Ángel D’Elía, Aristóbulo del Valle, Av. Pdte. Perón, Haedo, Sarmiento, Alberdi y nuevamente las vías del ferrocarril San Martín.

Más adelante, la zona se extendió desde la calle Aristóbulo del Valle hasta la calle Pardo (límite con la localidad de Bella Vista) y por esta retoma su límite de las vías del tren. La irrupción de estas viviendas multifamiliares de elevada altura y densidad dentro de un tejido situado en las antípodas de sus características, se tradujo en un cambio abrupto perceptible a nivel funcional. Esto es, en una mayor presión sobre los servicios sociales y de redes, de circulación del automotor individual y un fuerte desarrollo en extensión de los ya mencionados ejes comerciales de la ciudad. La consecuencia es, en ciertos casos, el desplazamiento de comercios hacia la periferia por los mayores costos de permanencia en el lugar, determinados por el incremento especulativo de los valores del suelo (tanto residencial como comercial) y los alquileres, íntimamente vinculados a estas fluctuaciones. (Ver ilustración 3).

Hay que adelantar en este punto, que la calidad de los servicios prestados en la zona se ajustaba a la densidad real que hasta entonces existía, pero que no estaban actualizados a la potencialidad de crecimiento que la norma vigente estipulaba. Esto determinó, tal como ocurría en otros centros urbanos de la metrópolis, una presión terminante sobre la calidad de las prestaciones básicas, sobre todo de agua potable, electricidad y gas.

\section{Evaluación de las tipologías edilicias}

La normativa vigente aplicable en el área de estudio emerge del Decreto-Ley 8912/77 de Ordenamiento Territorial y Uso del Suelo de la Provincia de Buenos Aires. Las demás disposiciones provinciales y ordenanzas y normativa complementaria municipal se ajustan a la misma y entre éstas encontramos: Ordenanza 448/79; sus modificatorias (Ordenanzas 278/86 y 237/87, y normas complementarias sobre cocheras, viviendas de encargado).

La norma mencionada, en especial la Ordenanza municipal 448/79 -cuya jurisdicción específica es la encargada de espacializar el modelo impuesto desde la norma superior-, determina para la zona denominada "Microcentro (en donde se inserta el área de estudio) los siguientes indicadores numéricos para obra nueva entre medianeras: FOS 0,6 y FOT: 3 . En cuanto a nuevos fraccionamientos, dado que la zona microcentro se trata de una trama cerrada (con la excepción de unos contados 
lotes vacantes), tal disposición al presente, no es aplicable y los índices numéricos se aplican directamente sobre el parcelario viejo. ${ }^{3}$

\section{Ilustración 3}

Demarcación de la zona microcentro (sombreada en verde pastel),

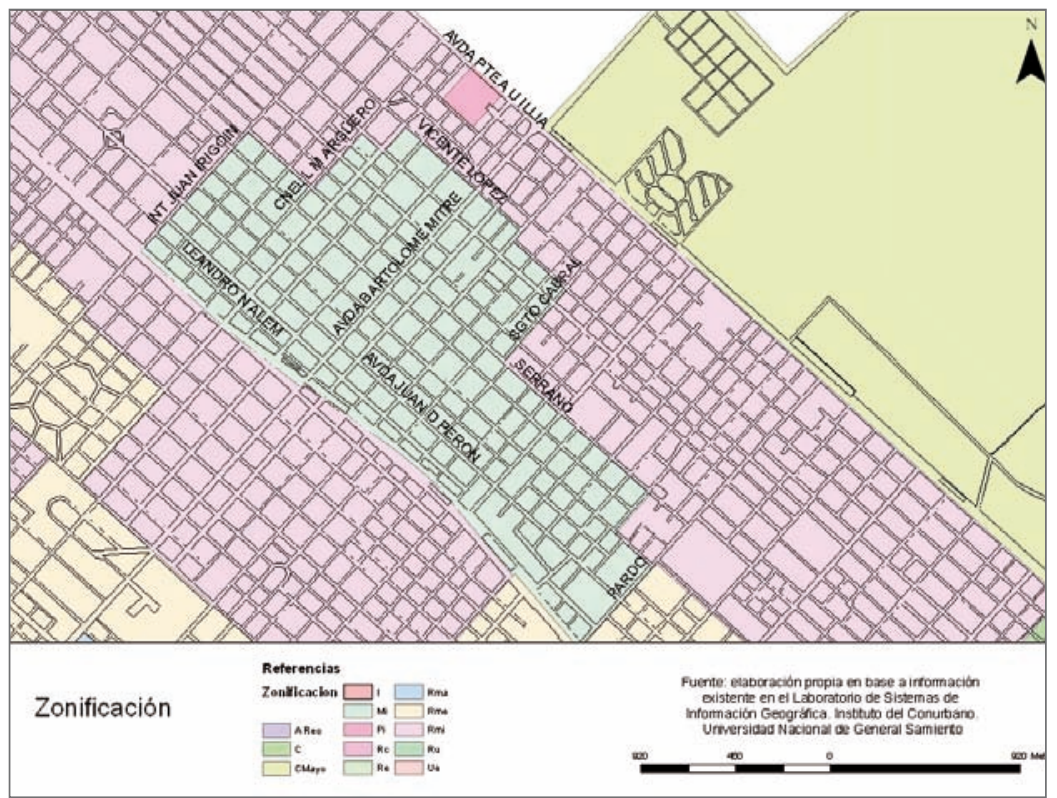

Fuente: Elaboración propia en base a información del LabSIG-UNGS.).

Los edificios en torre de la zona, con alturas que sobrepasan los 20 pisos, se caracterizan por la definición de una tipología propia, con edificaciones de perímetro libre, con planta baja destinada en su totalidad a espacios comunes. En algunos casos (que llamaremos tipo “ $A$ ”), los balcones son de superficies generosas y protuberantes; en otros (que denominaremos tipo "B") no hay balcones y las ventanas son levadizas y con una fuerte dotación de huecos para la instalación de acondicionadores de aire. (Ver imagen 4)

El ajardinamiento de la entrada es un rasgo marcado muy especialmente en las torres del tipo "A"; en el caso de las "B" se privilegia la dotación de cocheras a nivel de planta baja cuando es posible. La cesión de parte del frente al espacio público no reconoce un patrón común en todos los casos, ya que varía mucho de acuerdo a la

Asimismo, los indicadores para nuevos fraccionamientos de tierra son: lado mínimo: 25 metros; superficie mínima: $750 \mathrm{~m} 2$ y un ancho mínimo de calles: 15 metros.A los mencionados parámetros de obra nueva se aplican los beneficios en premios sobre el FOT hasta el 70\%, de cumplirse las condiciones dispuestas en el Decreto-Ley 8912/77: separación de medianera mayor a cuatro metros, menor índice de ocupación del suelo del establecido, cesión al espacio público, por retiro de fachada, englobamientos que superen el lado mínimo dispuesto para la zona, entre otros. 
torre y su ubicación. Esto es, si es un caso de lote en esquina o en el centro de la cuadra, si se ha dado espacio generoso a las cocheras, las superficies necesarias para las maniobras de ingreso a los estacionamientos subterráneos, espacios comunes como gimnasio, salón de usos múltiples, etc. ${ }^{4}$

\section{Ilustración 4}

Los dos modelos de torres de mayor presencia en el área de estudio.
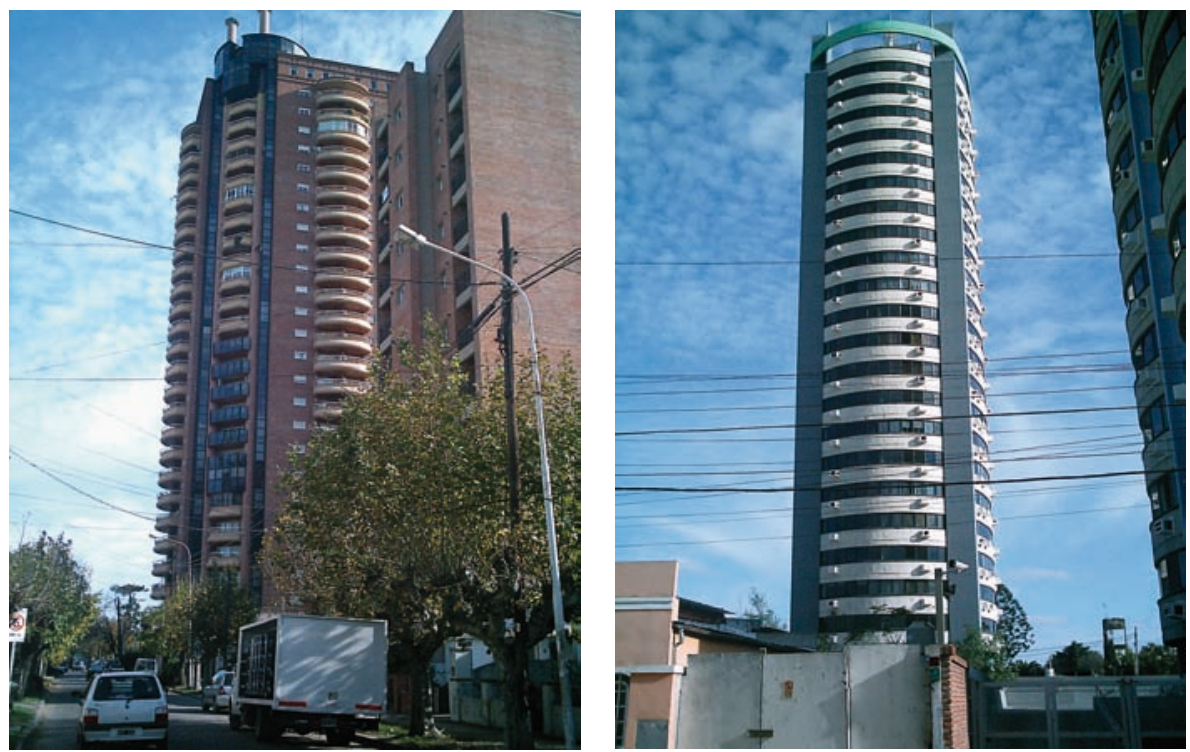

Fuente: Elaboración propia

La imagen tridimensional del área muestra volúmenes dominantes en la trama urbana sobre el tejido residencial de baja densidad. Con lo cual, como consecuencia derivada de la altura que alcanzan las torres, se proyecta una sombra que afecta sensiblemente el asoleamiento del tejido circundante.Asimismo, la mayor densidad implica un mayor volumen real y potencial de demanda de servicios, transportes y espacios públicos, y detona la expansión de desarrollos comerciales. (Ver ilustración 5).

El impacto de las nuevas edificaciones sobre la prestación de servicios sociales y de redes que abastecen la zona es alto, tanto en la provisión de agua y tratamiento

4 Asimismo, el ingreso de los servicios de redes está cuidadosamente articulado con las empresas para hacerlo de forma subterránea con el fin de no deslucir el aspecto externo de la construcción, distinto de lo que sucede con las edificaciones más antiguas. 


\section{de efluentes, ${ }^{5}$ como en los servicios educativos ${ }^{6}$ y sanitarios ${ }^{7}$ y también en la oferta de transporte público. ${ }^{8}$}

\section{Ilustración 5}

\section{Vista isométrica de la volumetría del área de estudio.}

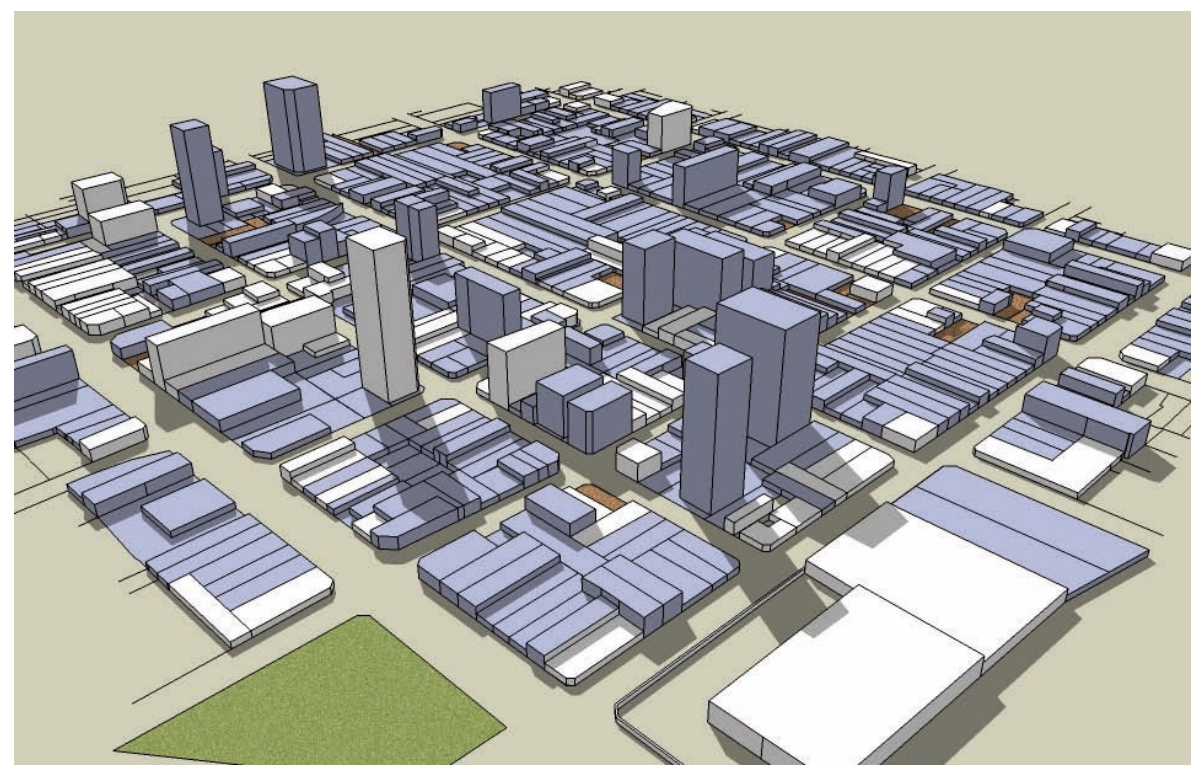

\section{Fuente: Elaboración propia.}

5 Luego de la rescisión del contrato de concesión a la anterior prestadora Aguas del Gran Buenos Aires S. A. en julio de 2006, la empresa estatal Aguas Bonaerenses S. A. toma a su cargo la provisión del servicio en la denominada Región Metropolitana Oeste. Esta región cuenta con sólo 170 mil cuentas de agua potable, a las cuales se abastece por medio de 188 pozos y una producción de ocho millones de metros cúbicos mensuales. En cuanto al aspecto del saneamiento, su oferta es únicamente para 172 mil viviendas con siete plantas depuradoras para toda la región. (Aguas Bonaerenses S.A., s.f.)

Dentro del área de estudio no hay ningún establecimiento educativo, pero sí encontramos una gran cantidad dentro del área de influencia determinada en $\mathbf{4 0 0}$ metros a la redonda. Los estatales se distribuyen en cuatro edificios y los privados son 16, destinados a los tres primeros niveles educativos y cinco a terciarios. (Dirección General de Cultura y Educación de la Provincia de Buenos Aires, s.f.)

7 En cuanto a las instalaciones de atención de la salud, se observa una importante presencia de consultorios médicos privados de varias especialidades y complejidades, pero ningún centro de atención público.

8 El área está servida por 38 ramales de colectivos atendidas por 17 líneas licenciadas a 13 empresas de transporte público de pasajeros. El servicio de ferrocarriles es prestado por las líneas San Martín y Urquiza. El último medio de transporte de pasajeros a considerar es brindado por taxis y remises: en la zona Microcentro sólo hay dos paraderos de taxis (estación San Miguel y en el cruce de Pardo y Av. Pdte. Perón, ambos fuera del área de estudio), pero varias agencias de remises dentro del área de estudio y de influencia, varias instaladas, incluso, en los locales habilitados a nivel planta baja de las torres o a escasos metros de ellas. 


\section{Una mirada desde el mercado del suelo}

El mercado inmobiliario de San Miguel vio modificada su dinámica a partir de 2004 , como resultado de este fenómeno de verticalización de la ciudad. Este proceso expulsó a muchas familias tradicionales de la zona frente a la presión ejercida sobre el suelo. Es posible encontrar una respuesta al fenómeno en la estratégica localización del Partido respecto de los partidos vecinos, sumado a la infraestructura existente y a los bajos precios de los lotes en relación con otras zonas del Conurbano bonaerense.

Si bien el discurso que justificaba el cambio en los patrones de crecimiento de la ciudad, pasando de un tejido de baja densidad, a uno denso, compacto, con edificación en altura, se escudaba en la resolución del problema de déficit habitacional que presentaba el Partido al principio de esta década, la población que accede al producto "torre" es externa a él. Mientras tanto, muchos de quienes tenían problemas habitacionales en el municipio no contaban con poder adquisitivo para acceder a la compra o al alquiler de estas unidades.

En breve lapso el precio por metro cuadrado se disparó a más de un $30 \%$. Teniendo en cuenta su dinámica inmobiliaria, el Microcentro se divide en dos sectores: desde la calle Irigoin hasta la Av. Mitre, y desde dicha avenida hasta la calle Pardo. Los precios del suelo en el primer sector comprendido entre Irigoin y Av. Mitre oscilan entre 300 y 450 dólares el m2, mientras que entre Mitre y Pardo, el valor se encuentra entre 420 y 600 dólares el m2. Pero dentro de esta área se puede realizar una diferenciación, respecto del emprendimiento ubicado en Av. Pdte. Perón y Saavedra, donde el precio del $\mathrm{m} 2$ varía entre mil 100 y mil 300 dólares.

San Miguel es ejemplo del modelo de ciudad impuesto por el Decreto-Ley $8912 / 77$ y de unas políticas urbanas locales que la sustentan. Por ello, y dada la jurisdicción sobre la cual se aplica el mencionado decreto-ley, resulta válida una "extrapolación" a otras centralidades suburbanas bonaerenses de nivel regional.

Por un lado, el caso muestra un cambio significativo en la configuración urbana en San Miguel, provocada por un nuevo patrón de crecimiento que es producto de la dinámica del mercado de suelo y que genera diferenciación socio-espacial y exclusión del mercado de los sectores más necesitados de respuestas habitacionales. Por el otro, muestra un gobierno local que enfrenta, con escasos recursos, el desafío de responder a la demanda de infraestructura y de equipamiento para absorber el crecimiento generado, cuyos beneficios son totalmente capitalizados por el sector privado.

\section{El proceso de gestión del territorio}

San Miguel, como tercer nivel de gobierno, recibe sus atribuciones del gobierno provincial, tal como lo establece el sistema federal de poder. Por ser un municipio de la Provincia de Buenos Aires, no tiene reconocida su autonomía como lo establece la Constitución Nacional de 1994 en su Art. I23, ya que la Constitución Provincial de 1994 no modifica lo establecido en materia de régimen municipal en la de 1933. La 
falta de autonomía implica un recorte importante en la capacidad del gobierno local para formular sus propias políticas públicas, ya que le quita la facultad de dictarse sus propias normas (Sabaya et ál., 2002).

En materia urbana, el ordenamiento territorial del municipio se rige por el Decreto Ley 8912 de la Provincia de Buenos Aires, que establece que: "Las comunas deberán realizarlo en concordancia con los objetivos y las estrategias definidas por el gobierno provincial para el sector y con las orientaciones generales y particulares de los planes provinciales y regionales de desarrollo económico y social y de ordenamiento físico". Estos planes provinciales y regionales no han sido objeto de la política provincial llevada hasta ahora.

Al momento de dictarse la Ley, los municipios debieron, en principio, determinar la afectación actual o futura a toda zona asignándole usos determinados y a estos asignarles extensión de ocupación del suelo (FOS), intensidad de ocupación del suelo por el uso y densidad (FOT). Además, tenían que definir subdivisión del suelo e infraestructura de servicios y equipamiento comunitario. Todos estos indicadores también están determinados en la misma ley.

En ella se estableció entonces, la obligatoriedad de la adecuación de la normativa municipal a la provincial. También, se determinaron plazos para las etapas de la planeación según las distintas situaciones en las que se encontraban los diferentes municipios en materia de ordenamiento territorial. Para esto se organizó una secuencia que incluía: delimitación de áreas, zonificación según usos, planes de ordenamiento municipal y planes particularizados.

En el caso de San Miguel, por Ordenanza 448/79 se aprobó el Código de Zonificación Preventiva, el que es definido por la ley provincial como un:

(...) instrumento técnico-jurídico tendiente a cubrir las necesidades mínimas de ordenamiento físico territorial, determinando su estructura general, la de cada una de sus áreas y zonas constitutivas, en especial las de tipo urbano, estableciendo normas de uso, ocupación y subdivisión del suelo, dotación de infraestructura básica y morfología para cada una de ellas.

Este es un paso preliminar a dotar al municipio de un Plan de Ordenamiento Municipal que, de acuerdo a lo establecido por el Art. 80:

(...) organizará físicamente el territorio, estructurándolo en áreas, sub-áreas, zonas y distritos vinculados por la trama circulatoria y programando su desarrollo a través de propuestas de acciones de promoción, regulación, previsión e inversiones, mediante métodos operativos de ejecución en el corto, mediano y largo plazo, en el cual deberán encuadrarse obligatoriamente los programas de obras municipales, siendo indicativo para el sector privado".

Este paso posterior no fue desarrollado hasta la fecha, por lo cual todo el desarrollo urbano del municipio sigue teniendo como principal instrumento de planificación urbana el Código de Zonificación Preventivo de fines de los setenta. Este ins- 
trumento, según la propia normativa, cubre las necesidades mínimas de ordenamiento territorial. La falta de adecuación de dicho Código a las nuevas situaciones generadas en el desarrollo urbano fue resuelta en parte por distintas ordenanzas convalidadas por la Provincia, como la de extensión de la zona Microcentro (Ordenanza 279/86) y la de viviendas mancomunadas (237/87).

También se cuenta con la posibilidad de autorizar proyectos, según lo establecido por la ley provincial en su Art. 102 (Decreto-Ley I0 I28/83):

Cuando el interés público lo requiera, el Poder Ejecutivo podrá regular, mediante Decreto, la autorización de proyectos referidos a situaciones particularizadas o zonas o distritos determinados, aún cuando no se satisfagan algunos de los recaudos o indicadores establecidos en la presente ley (...). El organismo provincial o municipal proponente deberá elevar la propuesta acompañada de los estudios que la fundamentan.

La estructura para gestionar el planeamiento urbano también está establecida en la ley provincial en su art. 74. "Los municipios contarán, dentro de la oficina de planeamiento, con un sector de planeamiento físico que tendrá a su cargo los aspectos técnicos del proceso de ordenamiento territorial del partido".

El organigrama municipal da la pauta del lugar que ocupan las distintas funciones referidas al territorio. Al planeamiento le corresponde la categoría de Dirección dentro de una Secretaría que moviliza principalmente los fondos provenientes de niveles superiores de Gobierno. Entonces, el marco legal provincial encuadra a sus municipios respecto de sus facultades dentro de una autarquía, es decir, pueden administrarse por sí mismos, pero dentro de normas que le son impuestas desde el nivel superior.

En lo que respecta al desarrollo urbano, esto tiene algunas consecuencias como son las de estar limitados al marco legal provincial para dictarse sus propios instrumentos (normativos, de planificación, de financiamiento, de promoción y desarrollo y de gestión del suelo). Esta limitación, sin embargo no significa que no se tengan competencias para promover el desarrollo urbano.

Se mostró que existen instrumentos que no han sido desarrollados en San Miguel, y se puede comprobar que han sido y están siendo convenientemente desarrollados y "agiornados" por otros municipios de la Provincia" para responder a los nuevos desafíos que enfrentan los gobiernos locales. Es de destacar que incluso estos planes son promovidos desde la Provincia a través del Ministerio de Economía y Producción de la Nación a través de la Unidad de Preinversión con financiamiento del Banco Interamericano de Desarrollo.

9 Plan de Desarrollo Estratégico de Morón, Plan de Ordenamiento y Desarrollo Urbano de Malvinas Argentinas, Plan Estratégico de San Fernando, Plan Urbano Ambiental de Moreno, entre otros. 


\section{Evaluación del modelo implementado}

El modelo de territorio al que respondía esta ley provincial coincide con el paradigma de planificación centralizada y tecnocrática del momento, basada en la idea de un urbanismo científico que facilitaba el diagnóstico y fundamentaba las acciones sobre la ciudad. El poder público municipal tuvo la función de planificar su territorio en base al control sobre la acción de los agentes privados a través de disciplinas de uso de suelo, vía zonificación, en línea con la normativa provincial. El resto del desarrollo urbano, inversiones en transportes, sistema viario, infraestructura y equipamientos públicos, dadas las limitaciones presupuestarias de los mismos, quedó básicamente en manos de la inversión del nivel provincial y (o) nacional.

La nueva forma de gerenciar los problemas interjurisdiccionales tuvieron su modelo palpable en la creación del $\mathrm{CEAMSE}^{10}$ y el Mercado Central, dos empresas autárquicas que se autofinancian (Abba, 2006). A nivel local, al municipio restaba avanzar con el Plan de Ordenamiento Municipal y los Planes Particularizados, que como ya se mencionó, no se desarrollaron hasta el momento.

Estas formas de encarar el desarrollo urbano, los instrumentos y las maneras de gestionar el territorio fueron cambiando, con el fin de dar respuesta a los viejos déficit heredados y a los nuevos desafíos ambientales y económicos (reducir desigualdades, austeridad de recursos escasos, productividad e innovación en los nuevos contextos económicos). A la vez se incorporan nuevas temáticas (la participación, el nuevo rol de los gobiernos locales).

También se comenzó a evaluar la articulación entre la planificación y la gestión por un lado, y entre la ciudad como totalidad y sus partes, por el otro. Surge, además, la idea de que la planificación urbana debe contar con instrumentos flexibles que permitan orientar las acciones en el mediano y largo plazo y también atender la coyuntura. Esa flexibilidad permite actuar ante una realidad dinámica y un contexto signado por conflictos de intereses entre los distintos actores involucrados.

Ya no se piensa en un proceso de planificación urbana que culmina con un Plan Director omnicomprensivo y exhaustivo surgido desde los organismos técnicos municipales, sino que se planifica desde una noción más instrumental basada en una estrategia urbana que admita posibles cambios. Es decir, se tratan de establecer lineamientos generales que orienten hacia un modelo de ciudad consensuado de forma participativa (Reese, 2006).

El Municipio de San Miguel en este contexto de cambios en la orientación de la planificación urbana continuó con el mismo esquema legislado por la Provincia, pero incompleto, aunque la realidad imponía nuevas presiones sobre el territorio y el desarrollo económico y social. En el área central comenzó un proceso de obsolescencia económica (Rojas, 2005), que implicó cambios parcela a parcela en los usos 
del suelo con incremento de las actividades comerciales y de servicio y densificación en altura.

Los primeros edificios que superaban los cinco pisos se emplazaron hace casi cinco décadas en el Microcentro. El comienzo de la construcción de torres en la localidad de Muñiz data de unos veinte años atrás con la construcción de una serie de torres en la calle Pardo entre D'Elía y Av. Perón y un conjunto de monoblocks frente al primer gran supermercado del Partido también sobre Av. Perón. Todos estos erigidos en una zona que no contemplaba los indicadores correspondientes a este tipo de emprendimiento. La presencia de estos edificios en un área de vivienda unifamiliar quedó convalidada por la Ordenanza 279/86. Con ella se extendió hasta la calle Pardo la zona Microcentro, aún cuando el área original no se había densificado y mostraba excepcionalmente edificios de más de dos plantas.

La sanción de la ordenanza no tuvo relevancia durante los siguientes 20 años, en los que se verifica un crecimiento moderado de la construcción. Esta tendencia cambia en el período -2003-2007 en el que se incrementa notablemente, tanto en el área original como en la extendida, siempre de forma aislada y discontinua.

La presencia de las torres de altura muy contrastante con la morfología del área, responde a los premios que otorga la Ley 8912 (de un 70\% sobre FOT y densidad y altura libre), o a las interpretaciones de la norma, que permite cierto grado de discrecionalidad. Pero, el hecho de que muchas de las obras hayan carecido de carteles de obra que indicaran expediente municipal aprobado, responsable de la construcción, del proyecto, etc., fue alimentando la inquietud y protestas de los vecinos. Ellos veían en algunas torres no sólo un producto inmobiliario que atentaba contra su forma de vida en el barrio, sino también un negocio que aparentemente no tenía sustento en ninguna normativa.

Durante estos años de expansión rápida del mercado inmobiliario expresado en el producto "torres", la inquietud vecinal llevó a la formación de comisiones y a distintas expresiones sociales y reclamos. La intención era mostrar al municipio su preocupación por la capacidad de soporte de la infraestructura de servicios ante el aumento de la densidad y la morfología urbana resultante de la implantación inorgánica de las torres (Ordenanza 3/2008).

Esto movió al gobierno municipal a dar sanción de la Ordenanza 3/2008. Con la misma suspendió por un plazo de hasta 180 días corridos los procedimientos para iniciar la aprobación de planos, ampliaciones, proyectos o permisos urbanísticos para la construcción de edificios que excedieran la planta baja y cinco pisos en parcelas ubicadas en la zona Microcentro.

Además, creó una Comisión Consultiva Municipal para la planificación urbana del distrito.

Esta estaba conformada por un máximo de 15 miembros de distintos niveles del gobierno local y sectores sociales. La misión era proponer los principales lineamientos y recomendaciones para confeccionar un nuevo Código de Planeamiento Urbano y especificar criterios a considerar en lo que respecta a la construcción de edificios en altura que excedan los cinco pisos. 
De alguna manera esta era una buena iniciativa para comenzar con las discusiones entre autoridades locales y de distintos niveles con injerencia en el territorio, distintos sectores socioeconómicos y culturales, organizaciones sociales, las universidades de la zona y vecinos interesados. El proceso podría servir para definir una estrategia de crecimiento y desarrollo urbano en función a un modelo de ciudad consensuado, de objetivos priorizados y acciones viables consensuadas que pudieran emprenderse y que se condensaran en un plan urbano, pero el plazo adjudicado no lo permitía.

El concepto fue más bien incluir algunas opiniones y lineamientos para la confección de un Código de Planeamiento Urbano, un instrumento operativo, que reemplazaría al Código de Zonificación Preventiva. Con esto tampoco se avanzaba en los dos pasos faltantes que establece la Ley provincial 8912, Plan de Ordenamiento Urbano y Planes Particularizados.

\section{La necesaria formulación de un plan urbano}

El proceso para el diseño y la sanción de un plan urbano en la actualidad implica la definición de una dirección global a la que se ajustarán las acciones, tanto públicas como privadas. Es decir, se intenta definir en el presente lineamientos básicos que pretenden alcanzar objetivos que tendrán repercusión en el futuro y que serán también el marco de referencia para resolver problemas actuales. Esto significa que es necesario desarrollar un proceso de planificación que tendrá como culminación una estrategia abierta a posibles cambios (pues no consiste en una predicción cerrada de escenarios "ciertos" de la evolución de la ciudad) y acompañada de proyectos urbanos que sintetizan esa estrategia (Reese, 2006).

Los planes urbanos, entonces, materializan los lineamientos generales del pacto urbano, producto del consenso entre los diferentes actores (públicos y privados) que participan en el mismo. Dentro de éste, se concretan proyectos que operan sobre aquellos problemas que permitan la modificación de la estructura urbana existente. Estas características hacen del plan urbano un instrumento de gestión y de negociación, el Código es sólo uno de los instrumentos normativos que componen dicho plan .

Por otra parte, se puede analizar la apertura a la participación que significó la Comisión. Cuando se habla de proceso participativo, de la obtención de consensos para elaborar un plan que refleje la ciudad “deseada”, se está teniendo en cuenta que la ciudad es producto de procesos sociales que generan estructuras que la reproducen tal cual es, en toda su complejidad. Por lo tanto, para poder hacer un diagnóstico y proponer una estrategia, es necesario llevar a cabo un proceso que permita recomponer las miradas parciales que den cuenta de una visión integral de la ciudad y su problemática. Al mismo tiempo, se necesita interpretar los comportamientos y relaciones entre los distintos actores sociales involucrados y los conflictos que se puedan derivar al intentar generar cambios en las estructuras consolidadas. 
De esta forma, la participación de representantes de distintos niveles de gobierno y de organizaciones sociales, económicas, religiosas, medios locales de difusión, así como el apoyo técnico de las universidades que se localizan en la zona, desde las primeras instancias, permite captar los distintos puntos de vista de estos actores sobre la problemática del municipio. También evaluar sus grados de poder, anticipar apoyos y conflictos, e involucrarlos en la toma de decisiones, la difusión y el liderazgo del proceso, con el fin de construir viabilidad política y social. Se trata de una participación multisectorial y multinivel.

El rol del gobierno local, en este proceso, es el de favorecer la actuación de los diversos actores sociales en la construcción de la ciudad. Su papel es garantizar que las estrategias de intervención expresen los mayores niveles de consenso, sin delegar su responsabilidad rectora en la definición de políticas urbanas. Como se ve, es un proceso mucho más complejo y abarcativo que el propuesto con la Comisión.

Lamentablemente, un proceso de este tipo todavía no ha podido llevarse a cabo en su amplitud y complejidad, a pesar de que permitió mostrar una cierta apertura del gobierno local por incorporar algún tipo de instancia participativa en la definición de sus políticas urbanas.

Contrariamente a lo esperado, el resultado del proceso participativo no fue exitoso en cuanto a la obtención de un producto. El trabajo en la Comisión llevó a una propuesta de la Secretaría de Obras Públicas que proponía rever los términos de la zonificación vigente y los indicadores urbanísticos y usos, en el Microcentro. También, como propuesta global, proponía proteger un rol deseado a nivel municipal y provincial como cabecera de Partido y como potencial cabecera regional.

Esta proposición fue rechazada por un lado, por muchos de los participantes de la Comisión, por no reflejar sus opiniones vertidas en la misma y por lesionar los derechos adquiridos de los propietarios de la zona Microcentro. Con esto se refiere a las expectativas de construir futuras torres. Por el otro, el proyecto de ordenanza no obtuvo la aprobación del Concejo Deliberante con el mismo argumento sobre los derechos adquiridos.

Profundizar sobre la legitimidad de los derechos adquiridos no es tema de este trabajo, pero aquí se muestra reflejado un problema que enfrenta a los vecinos. Por un lado, los que dicen tener derechos adquiridos por haber construido en un barrio con características de baja densidad (de acuerdo con el Código de Zonificación Preventiva) y por el otro, los derechos adquiridos “en expectativa”. Es decir, los invocados por los propietarios de suelo que pretenden construir en el futuro en formato torre, de acuerdo con la normativa posterior que se los permite.

Hay que destacar que el gobierno local, por otra parte, también tuvo una apertura a la idea de participación con la aprobación y puesta en práctica del Presupuesto participativo (Ordenanza 40/2008). Este instrumento permite un diálogo entre los vecinos y el municipio sobre la problemática de los barrios y también consensuar y priorizar algunas soluciones. En la zona de estudio funciona una asamblea barrial denominada Muñiz Norte. 
El proceso de densificación del área centro extendido se da entonces en un marco donde aparecen por un lado, las torres en el mercado como respuesta a la demanda de un sector social específico, propuesta que es vista por el sector público como una forma de atraer inversiones privadas. Por otro lado, las luchas de los vecinos en contra de ese producto ajeno a muchos barrios con tradición de vivienda unifamiliar en distintos lugares de diferentes ciudades que va generando un discurso compartido. Además, aparece un discurso académico que plantea una idea de ciudad física y socialmente integrada según un planeamiento que articule actores públicos y privados, de modo que la densificación responda a una idea de ciudad para todos, más equitativa y más eficiente. Este también va ganando espacio en el discurso de los vecinos y en los medios de comunicación.

En línea con el contexto general, el desarrollo urbano del área de estudio, y más ampliamente en la zona Microcentro, presenta redes de actores en conflicto. La articulada en función de un desarrollo urbano basado en el producto torre, los vecinos del área -que en algún momento tuvieron una actuación colectiva- $y$ algunas instituciones que ven con preocupación un avance de la densificación de áreas de baja densidad sin un plan que integre las torres a un proyecto de ciudad.

\section{Nuevos instrumentos de desarrollo urbano}

Uno de los déficit que tienen los municipios de la Provincia de Buenos Aires se refiere a su poco margen de maniobrabilidad en cuanto a dictarse sus propios instrumentos de desarrollo urbano. Pero, también se mencionó que esto no impidió en muchos casos implementar planes urbanos que atendieran a la vez a la normativa y a los nuevos desafíos de la planificación y gestión local. Estos fueron asociados, en general, a una visión estratégica de mediano y largo plazo que además pudiera atender la coyuntura, cuestión vital para la gestión política.

Es decir, que una buena cantidad de municipios de la Provincia ya advirtieron que la persistencia en un modelo de planeamiento basado en el zoning y la normativa ad hoc no se adapta a la compleja realidad urbana actual. Algunos de ellos incluso han incursionado en iniciativas tendientes a diseñar soluciones consensuadas a nivel regional para la solución de problemáticas comunes al área (Red de Planificación Estratégica de Gobiernos Locales).

Ese modelo tradicional de desarrollo implica la necesaria intervención del Estado central en la financiación de obras de infraestructura y equipamiento. Actualmente, con la descentralización de funciones, los municipios enfrentan la delegación de responsabilidades sobre distintos aspectos del desarrollo urbano (físico, social, económico, ambiental). En este nuevo marco, afrontan la necesidad de articular el crecimiento de la ciudad y el desarrollo local sin contar con instrumentos de financiación propios para direccionar el progreso.

Ante esta nueva situación, se encontrarían en la disyuntiva de no crecer, o facilitar el desarrollo a través del mercado según sus leyes. Pero, esta es una falsa 
disyuntiva que surge de un modelo de gestión no adecuado a la realidad socioeconómica y política y a las nuevas formas de planificar el desarrollo urbano. El caso de las torres en San Miguel permite poner en relieve algunos de los efectos negativos de una gestión de tipo tradicional que facilita un desarrollo urbano ligado a los intereses del mercado:

- En el aspecto social: foros vecinales de protesta; movilización de vecinos; judicialización de los proyectos (caso del Club San Miguel) y desplazamiento de población residente.

- En el aspecto económico: concentración de inversiones privadas en un área con aumento de precio del suelo; creciente necesidad de equipamiento y servicios que deben ser financiados exclusivamente con inversión pública (no local); falta de instrumentos locales de financiación de obra pública, de redistribución de los beneficios de la urbanización y de articulación de gestión público-privada.

- En el aspecto fisico: déficit de servicios (agua); déficit de equipamiento (sanitario, escolar, espacio público); congestión vehicular; aumento de los usuarios de transporte público sin aumento de servicios; fragmentación espacial.

- En el aspecto político: crisis de los marcos regulatorios vigentes; falta de integralidad en la planificación urbana; falta de coherencia y visibilidad de las acciones que realiza el gobierno local en el territorio; falta de un horizonte de crecimiento consensuado que posicione a dicho gobierno como actor protagónico en el marco de un Estado con fuerte presencia articuladora, fiscalizadora, orientadora y ejecutiva; aislamiento respecto de los gobiernos de la región que conforman la Red de Planificación Estratégica de Gobiernos Locales; falta de un instrumento de negociación política y de orientación de las inversiones públicas y privadas en el territorio.

Ante este escenario, la pregunta que queda abierta es: ¿por qué se siguen construyendo torres en municipios como San Miguel, donde persiste el conflicto y la incertidumbre sobre la capacidad de respuesta de las infraestructuras y los servicios urbanos? Todo lo señalado muestra que cuando un municipio opera un cambio drástico en su estructura urbana que pone en conflicto a la ciudad como bien de uso (que implica a los vecinos residentes) vs. la ciudad como bien de cambio (la de los inversores locales o extra locales), se hace presente como nunca la necesidad de consensuar un nuevo contrato urbano. Este debe definir el nuevo perfil de ciudad deseada.

Este contrato requiere de la participación de toda la comunicad interesada, no debería limitarse a un número restringido de actores involucrados en el desarrollo urbano. No debería tampoco ser limitado a un sector de la ciudad y a un tema específico. La ciudad opera de forma integral, no por fragmentos aislados, y es un sistema interrelacionado. Por esto, por ejemplo, un aumento de densidad poblacional de un sector implicará la necesaria oferta de equipamiento (de salud, educativo, espacios verdes) y servicios (agua, cloacas, transporte) que la planificación deberá prever, tanto en su dimensión física como en la disposición de los recursos necesarios.

Tampoco puede limitarse a una morfología urbana dictada por las modas del mercado inmobiliario: la densificación, si es necesaria, tiene muchas formas de resolución, algunas más amigables que otras con la ciudad existente (Reese, 2006). Pero 
¿cómo se lograría un quiebre en el modelo de planificación y gestión actual que ponga a San Miguel en sintonía con los municipios vecinos que ya han operado el cambio?

Podemos considerar que la voluntad de gestión puede definir el tipo de política urbana a seguir orientándose hacia la permanencia dentro de las formas tradicionales de gestión o hacia formas que Gomá y Blanco (2002) definen más cercanamente al concepto de governance. Es decir, la gestión apela al poder de representatividad del gobierno local para hacer una lectura de la situación y decidir sobre los mecanismos más adecuados para desarrollar el distrito de acuerdo al interés general. Esta lectura puede desembocar en una decisión de implementar algún tipo de gestión que incluya diferentes miradas e intereses en la definición de las políticas urbanas.

Pero se podría considerar también, que debido a que la planificación que apunta a la governance implica cambios sustanciales en las estructuras locales de gestión que pueden ser reacias a dichos cambios, algunos hechos podrían influir y hasta forzar una lectura en el sentido de una gestión menos jerárquica. Circunstancias como la movilización de distintos sectores en torno de la problemática urbana podría constituirse en una ventana de oportunidad para que el gobierno local dé cuenta de la necesidad de implementar formas de gestión que permitan articular esos actores sociales, económicos y políticos.

De esta manera se podrían abrir espacios de planificación participativa tendientes a dar respuestas a demandas diversas con una solución consensuada de políticas urbanas. Aunque siempre permanece latente la preocupación por las acciones de aquellos actores con mayores cuotas de poder que puedan coartar tales ensayos cuando se presenten contrarios a sus intereses de grupo. La condición para que este proceso sea efectivo es contar con una "una sociedad civil en la que los individuos gocen de igualdad como ciudadanos, $y$ en la que los intereses privados encuentren una expresión organizada" (Maintz, 200I).

El municipio de San Miguel está en este momento encarando un presupuesto participativo, creando redes con la sociedad que le permitirán tener un acercamiento a la problemática socio-territorial desde la perspectiva de los vecinos. También, ha convocado a una comisión para la revisión de los indicadores urbanísticos del micro-centro que aunque no concluyó en el producto esperado, fue una experiencia que procuraba un consenso sobre una fracción del territorio.

Como antecedentes existen, asimismo, los encuentros que tuvieron lugar durante los últimos años por las preocupaciones de los vecinos respecto a los desarrollos inmobiliarios de la zona, algunos de los cuales fueron discutidos en dos Jornadas de la Universidad Nacional de General Sarmiento a las que concurrieron funcionarios municipales. Estos antecedentes podrían estar creando las condiciones de oportunidad para desarrollar un Plan Urbano que viabilice la transformación urbana a través de una nueva relación entre la sociedad civil y el Estado, que permita consensuar el modelo de ciudad deseada. 


\section{Bibliografía}

Abba,A. (2006). “Formar institucionalidad metropolitana en Buenos Aires”. Propuesta de gestión para el AMBA. Café de las Ciudades, revista electrónica, año 5, $\mathrm{n}^{\circ}$ 48, octubre.

Aguas Bonaerenses S.A. (s.f.). La Empresa. Recuperado el 3 de julio de 2009, de sitio web de Aguas Bonaerenses S.A.: www.aguasbonaerenses.com.ar/la-empresa. htm.

Atlas Ambiental de Buenos Aires. (2009). Centralidades Urbanas. Recuperado el 20 de julio de 2009, de sitio web del Atlas Ambiental de Buenos Aires: www.atlasdebuenosaires.gov.ar/aaba/index.php?option=com_content\&task=view\&id = I I 8 \&ltemid=49\&lang=es

Borja, J. y Castells, M. (1998). Local y global. La gestión de las ciudades en la era de la información. Madrid:Taurus.

Ciccolella, P. (1999). “Globalización y dualización en la Región Metropolitana de Buenos Aires. Grandes inversiones y reestructuración socioterritorial en los años noventa”. Eure, 25 (76).

Covelia S.A. (2009). Municipios. Recuperado el 20 de julio de 2009, de sitio web de Covelia S.A.: <www.covelia.com.ar/municipios.htm>.

De Mattos, C. (200I). “Metropolización y suburbanización”. Eure, 27 (80).

Dirección General de Cultura y Educación de la Provincia de Buenos Aires. (s.f.). Mapa escolar. Recuperado el 3 de julio de 2009, de sitio web de la Dirección General de Cultura y Educación de la Provincia de Buenos Aires: <http://mapaescolar.ed.gba.gov.ar/examples/servlets/pr I7/mapa\%20escolar/index.htm>.

Distrito IV - Colegio deArquitectos de la Provincia de Buenos Aires. (2008). Jornada de Estudio y Análisis de los Códigos de Ordenamiento Urbano y Planificación. Recuperado el 20 de julio de 2009, de sitio web del Distrito IV - Colegio de Arquitectos de la Provincia de Buenos Aires:<http://www.capbaiv.org.ar/component/ option.com_frontpage/ltemid, I 58/limit,8/limitstart, | 6/>.

Gomà, R., \& Blanco, I. (2002). “Gobiernos locales y redes participativas: retos e innovaciones”.VII Congreso Internacional del CLAD sobre la Reforma del Estado y de la Administración Pública. Lisboa.

INDEC. (200I). Censo nacional de población, hogares y vivienda del año 200 I.

INDEC. (2003). ¿Qué es el Gran Buenos Aires? Buenos Aires: INDEC.

Maintz, R. (200I): “El Estado y la sociedad civil en la gobernanza moderna”, en Revista Reforma y Democracia, $\mathrm{n}^{\circ} 2 \mathrm{I}$, octubre, Caracas.

Miño, M., Borello, J., \& y Alsina, G. (2007). Diagnóstico ambiental preliminar del Partido de San Miguel. Los Polvorines: Universidad Nacional de General Sarmiento.

Municipalidad de San Miguel. (2009). Presupuesto participativo. Recuperado el 20 de julio de 2009, de la Municipalidad de San Miguel:www.msm.gov.ar/index_pp.php.

Randle, P. H. (1977). La ciudad pampeana. Buenos Aires: Oikos.

Reese, E. (2006). Evolución de la práctica urbanística argentina. Taller de Urbanismo III. Universidad Nacional de General Sarmiento: Malvinas Argentinas, 16 agosto, (presentación).

Rojas, E. con colaboración de Rodríguez, E. y Wegelin, E. (cols.). (2005). Volver al centro. La recuperación de áreas urbanas centrales. México:Alfaomega-BID. 
Sabsay D., García, M. C., Nápoli, A. y Ryan, D. (2002). Región Metropolitana de Buenos Aires. Aporte jurídico-institucional para su construcción. Buenos Aires: Fundación Ambiente y Recursos Naturales.

Schneier-Madanes, G. (1998). Buenos Aires: une métropole en projet. París: Urbanisme, 298, pp. I4-33.

Tella, G. (200I). Del suburbio a la post-periferia. Efectos una modernización tardía en la región metropolitana de Buenos Aires. Buenos Aires: Ediciones de la Facultad de Arquitectura, Diseño y Urbanismo de la Universidad de Buenos Aires.

Tella, G. (2003). La mutación de enclaves urbanísticos en Buenos Aires. Espiral, Estudios sobre Estado y Sociedad, vol. IX. Guadalajara: Universidad de Guadalajara, pp. 211-237.

Tella, G. (2005). Ínsulas de riqueza en océanos de pobreza... o el proceso de fragmentación territorial de Buenos Aires. Barcelona: Universidad de Barcelona; ScriptaNova revista electrónica de Geografía y Ciencias Sociales; vol. IX, n 194.

Tella, G. (2007). Un crack en la ciudad: Rupturas y continuidades en la trama urbana de Buenos Aires. Buenos Aires: Nobuko.

Torres, H. A. (200I). "Cambios socioterritoriales en Buenos Aires durante la década de 1990”. Eure, 27 (80). 